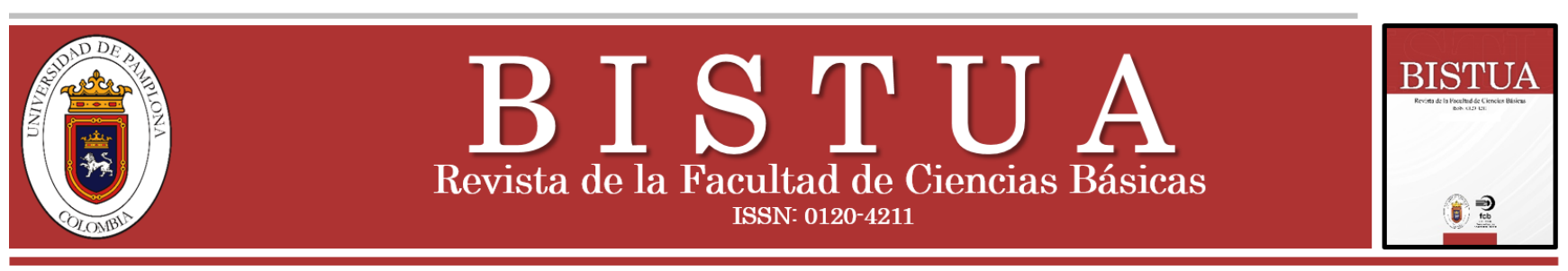

http://revistas.unipamplona.edu.co/ojs_viceinves/index.php/BISTUA

\title{
Correlación entre el flujo vehicular, el PM2,5 y variables meteorológicas, un estudio de caso al oriente de Bucaramanga (Colombia)
}

\section{Correlation between vehicular flow, pm2.5 and meteorological variables, a case study east of Bucaramanga (Colombia)}

\author{
Ligia Beleño Montagut ${ }^{a}$; Juan Diego Colegial Gutierrez ${ }^{b}$; Martha Lucia Barrera Perez ${ }^{a}$ \\ ${ }^{a}$ Universidad Autónoma de Bucaramanga, Colombia \\ ${ }^{b}$ Universidad Industrial de Santander, Colombia \\ Contacto: lbeleno@unab.edu.co
}

Recibido: Noviembre 30, 2020. Aceptado: Diciembre 27, 2020

https://doi.org/10.24054/01204211.v2.n2.2020.4438

\section{Resumen}

El problema de dispersión atmosférica de un contaminante es un tema complejo, donde variables como la velocidad del viento, la temperatura, la época del año, entre otros, contribuyen en mayor o menor grado al incremento o decremento de la concentración de las especies químicas. El objetivo de la investigación fue identificar la correlación entre variables climatológicas y la contaminación por los automotores (PM2,5 Y COV) en un punto de acceso a una institución ubicada al oriente de Bucaramanga (Colombia). Con relación a los datos del estudio, se utilizó un medidor manual y se registraron datos de PM2,5, Compuestos Orgánicos Volátiles (COV) y variables climatológicas de temperatura y humedad relativa para 26 días en la zona de interés durante 3 franjas horarias específicas del día, cada 5 minutos; además, se utilizaron los datos relacionados con la dirección y velocidad del viento de la estación Neomundo, según el documento del Instituto de Hidrología, Meteorología y Estudios Ambientales (IDEAM) para los años (2005-2009), por ser una zona similar en cuanto a la topografía. El análisis se realizó usando el software libre R (versión 3.5.3) y su librería OpenAir. Los resultados obtenidos muestran las correlaciones correspondientes del PM2,5, los COV, y las variables ambientales corroborando por una parte la influencia del tráfico vehicular en la concentración de PM2,5 principalmente debido a las motocicletas, y por otra parte la relación del PM2.5 con respecto a la Humedad relativa y la temperatura.

Palabras clave: atmósfera, contaminación, medio ambiente, calidad ambiental, indicadores ambientales

Palabras clave: atmósfera, contaminación, medio ambiente, calidad ambiental, indicadores ambientales

\section{Abstract}

The problem of atmospheric dispersion of a pollutant is a complex issue, where variables such as wind speed, temperature, time of year, among others, contribute to a greater or lesser degree to the increase or decrease in the concentration of chemical species. The objective of the research was to identify the correlation between climatological variables and pollution from motor vehicles (PM2.5 and VOC) at an access point to an institution located east of Bucaramanga (Colombia). Regarding the study data, a manual meter was used and data on PM2.5, Volatile Organic Compounds (VOC) and climatological variables of temperature and relative humidity were recorded for 26 days in the area of interest during 3 specific time bands of the day, every 5 minutes; In addition, the data related to the direction and wind speed of the Neomundo station were used, according to the document of the Institute of Hydrology, Meteorology and Environmental Studies (IDEAM) for the years (2005-2009), as it is a similar area in terms of to topography. The analysis was performed using free software R (version 3.5.3) and its OpenAir library. The results obtained show the corresponding correlations of PM2.5, VOCs, and environmental variables, corroborating, on the one hand, the influence of vehicular traffic on the concentration of PM2.5, mainly due to motorcycles, and on the other hand, the relation of PM2. 5 with regard to relative humidity and temperature.

Keywords: atmosphere, pollution, environment, environmental quality, environmental indicators

\section{Introducción}

El material particulado es de gran preocupación dado que está demostrado que en particular el PM2,5 produce efectos adversos sobre la salud [1]. No obstante, en Colombia los estudios relacionados con las concentraciones de PM2,5 son limitados dado que su medición no está disponible en algunas ciudades, "según los inventarios de emisiones 
realizados para los centros urbanos del país, el material particulado es emitido principalmente por la quema de combustibles fósiles, por el transporte automotor y el sector industrial. Se estima que aproximadamente el $80 \%$ del PM2,5 es generado por las fuentes móviles mientras que el $20 \%$ restante lo aportan las fuentes fijas" [2]. Por otra parte, se han hecho estudios que relacionan la emisión contaminante con PM10 y la salud como es el caso de Morales y Arias [3], donde se evidenciaron dos comportamientos relevantes: El primero de ellos es la relación entre morbilidad y emisión, y el segundo la relación entre morbilidad y concentración. También, Ramírez y otros [4], realizaron un estudio en Bogotá (Colombia) sobre el PM10 sugieren que "teniendo en cuenta que la fracción carbonosa de PM se ha relacionado principalmente con PM2.5, así como con los niveles de trazas de metales, se deben realizar más investigaciones sobre la distribución de PM2.5"

Un estudio realizado en Cuenca (Ecuador) por García [5], cuya zona urbana está caracterizada por el alto flujo vehicular, registran niveles altos en puntos de baja densidad vehicular, lo cual les sugiere que puede ser debido al tráfico lento en la zona y dado que los motores permanecen encendidos mientras los autos están detenidos. No se incluyeron variables meteorológicas en el estudio, pero proponen análisis respecto al material particulado y los parámetros que rigen el fenómeno de dispersión.

En Colombia, algunos de los trabajos acerca del análisis de emisiones de contaminantes en distintas regiones hacen referencia a la contaminación por flujo vehicular en zonas específicas, como es el caso de un estudio realizado en Cali, donde se desarrolló una investigación para estimar los Factores de Emisión (FE) de Monóxido de Carbono (CO), Dióxido de Carbono (CO2), Óxidos de Nitrógeno (NOX) e Hidrocarburos (HC) emitidos por los vehículos de la ciudad con el programa Mobile6, consideraron las liberaciones totales a la atmósfera de estos contaminantes, y se encontró que en el periodo de estudio (1996-2006) todas las emisiones se incrementaron [6]. El estudio realizado en Pamplona (N.S.) por Quijano y otros [7], utilizó un equipo de cromatografía de gases para identificar los hidrocarburos aromáticos policíclicos (HAP) presentes en el PM2,.5 del aire de Pamplona (extracto global y las fracciones); los resultados de los metales e hidrocarburos aromáticos policíclicos y los (HAP) encontrados en el PM2.,5 del aire de Pamplona los cuales concuerdan con un estudio previo en una zona netamente vehicular; y estos son principalmente emitidos de los tubos de escape del tráfico que es la principal fuente de PM2,.5 en esta ciudad. Los resultados del estudio sugieren que parte de la genotoxicidad mostrada por el aire de Pamplona, es ocasionada por los HAP encontrados, según evidencias que correlacionan la presencia de estos compuestos y el riesgo para la salud humana.

Por otra parte, En Manizales, se realizó una aplicación del modelo Gaussiano para determinar la calidad de aire de la ciudad y encontró que el 85,1 \% del CO2 generado se debe a la emisión de las fuentes móviles (diésel y gasolina) [8].
Con relación a la topografía, la dirección y velocidad del viento y el comportamiento de la dispersión de contaminantes, en el municipio de Socha, y en una zona simple ubicada en el Municipio de Sogamoso (dos zonas topográficas diferentes), Arrieta [9] hizo un estudio de partículas PM10 mediante la modelación con el Software AERMOD View y en su resultado identificó que la velocidad y dirección del viento son las variables meteorológicas que tienen un efecto directo en la dispersión de los contaminantes en el aire.

De acuerdo con Arciniegas [10], el monitoreo de partículas suspendidas en la atmósfera de las ciudades colombianas deben ser complementados con trabajos investigativos de partículas cada vez más pequeñas, siendo recomendable que los estudios relacionados con el material particulado tengan un seguimiento por parte de las autoridades ambientales y centros académicos, al igual que implementar estrategias de control para la utilización de combustibles limpios por parte de las autoridades colombianas, con el objeto de minimizar las emisiones de partículas por las fuentes móviles.

El documento del Ministerio de Ambiente y Desarrollo Sostenible [11] reporta que la emisión producida por las fuentes móviles varía según su aceleración, tipo de combustible y otras variables. Ya en el ámbito de la ciudad de Bucaramanga, según el inventario de emisiones de Fuentes Móviles de acuerdo al tipo de combustible, con información local publicada por el Área Metropolitana de Bucaramanga (AMB) [12]. Dichos resultados muestran que la mayor contribución de VOC es debida al uso de gasolina como combustible y el PM2,5 es mayor en automotores que usan diésel.

Según los datos de calidad de aire en Bucaramanga, desde octubre de 2018 el AMB realiza monitoreos diarios a través del Sistema de Vigilancia de Calidad del Aire 'Eolo' del AMB, mediante por cinco estaciones de medición de calidad del aire, una de ellas en el Instituto Caldas en Bucaramanga en la zona oriente según la Fig. 1, ubicada en condiciones topográficas similares a la zona de estudio (las demás estaciones de monitoreo se encuentran en distintas partes de la ciudad con condiciones topográficas muy diferentes), y según los resultados obtenidos por dicha estación de monitoreo, respecto a PM 2.5 se encontró en rangos "dañino a la salud de grupos sensibles" y "dañino a la salud", de acuerdo a los rangos del Índice de Calidad de Aire. Durante los días de medición se registraron niveles de concentración entre 41 y $82(\mu \mathrm{g} / \mathrm{m} 3)$, (el nivel máximo permisible de PM 2.5 para un tiempo de exposición de 24 horas es de $37 \mu \mathrm{g} / \mathrm{m} 3$ ) según información de la Alcaldía de Bucaramanga [13]. 


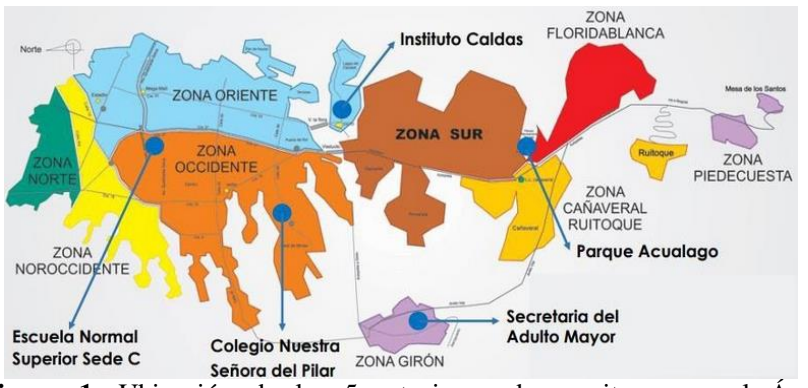

Figura 1. Ubicación de las 5 estaciones de monitoreo en el Área Metropolitana de Bucaramanga

Fuente: https://www.amb.gov.co/el-amb-realiza-monitoreo-diario-dela-tendencia-de-la-calidad-del-aire-en-bucaramanga-floridablanca-y-giron/

Los compuestos liberados a la atmósfera aún en bajas concentraciones pueden llegar a ser nocivos para la salud si son recurrentes, En los resultados del estudio de Rodríguez y otros [14] plantea que" las concentraciones de las sustancias químicas que componen al material particulado se encuentran asociadas con las fuentes generadoras"; por lo tanto, los aforos medidos para las tres franjas horarias pueden ser interpretados como indicadores de las cantidades de contaminante máximas emitidas. Esta dispersión depende de las características del entorno de la fuente de emisión (presencia de montañas, cambios en la temperatura, dirección del viento, etc.). Dichos compuestos pueden ser liberados por fuentes fijas o por fuentes móviles. El equipo de trabajo del Área Metropolitana de Bucaramanga AMB, de acuerdo al análisis de fuentes móviles por categoría vehicular en cuanto a la cantidad de emisión de Compuestos Orgánicos Volátiles (VOC) afirma que las motos 4T contribuyen en mayor cantidad y en segundo lugar los autos particulares. Las emisiones de PM2,5 más altas corresponde a la categoría Camiones, seguidamente motos $4 \mathrm{~T}$, Volquetas, Buses y Autos particulares [12].

Para el caso del presente estudio, no hay reportes con relación a proceso o equipos que generen emisiones en cercanías a la universidad (calderas, hornos, procesos de molienda, procesos de producción, entre otros), y de acuerdo a un estudio de la ciudad, por Rodríguez y otros [14], se encontró que los vehículos que utilizan combustibles fósiles, como los buses, vehículos y motos, son los principales generadores de contaminación atmosférica en el municipio de Bucaramanga. El objetivo es estimar la correlación entre variables climatológicas y la contaminación por los automotores en un punto de acceso a una institución ubicada al oriente de Bucaramanga (Colombia) de manera que se pueda contar con un estado base acerca de dicho comportamiento para posteriores estudios. Para el análisis de correlación entre las variables se utilizó el software libre R (versión 3.5.3) y su librería OpenAir [15].

\section{Materiales y métodos}

\section{Zona de estudio}

La ciudad de Bucaramanga, capital del departamento de Santander (Colombia) está localizada al nororiente del país y la zona urbana se encuentra dentro del Área Metropolitana de Bucaramanga (AMB) de la cual forman parte Bucaramanga, Floridablanca, Girón y Piedecuesta; se encuentra a 959 m de altura sobre el nivel del mar y sus pisos térmicos varían de acuerdo a su topografía: cálido $55 \mathrm{~km}^{2}$, medio $100 \mathrm{~km}^{2}$ y frío $10 \mathrm{~km}^{2}$. Su precipitación media anual es de $1.041 \mathrm{~mm}$ con una temperatura media de $23^{\circ} \mathrm{C}$. La división Político-Urbana de Bucaramanga corresponde a 17 comunas, y el trabajo aquí presentado se desarrolló en un punto específico de la comuna 12 (Ver Fig. 2)

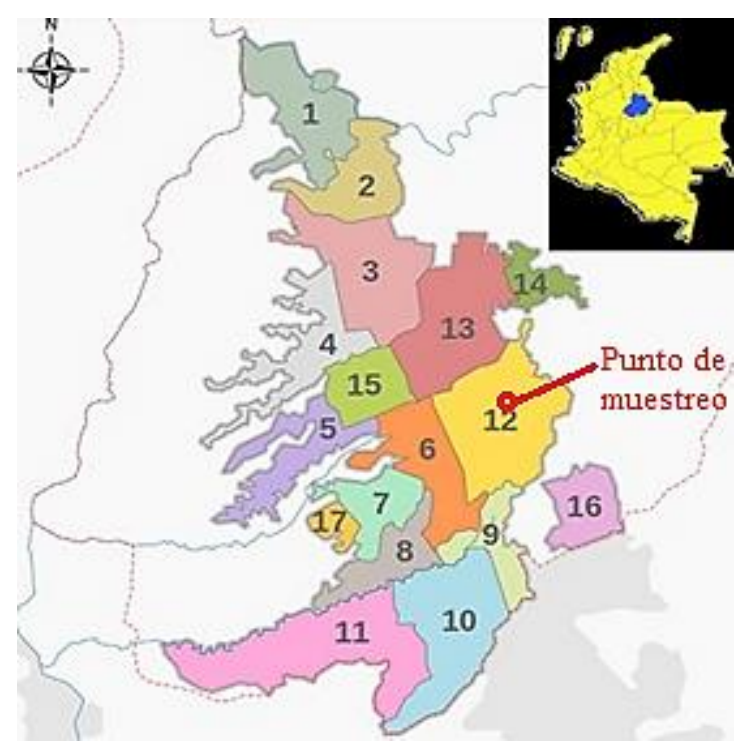

Figura 2. División Política-Urbana de Bucaramanga

Fuente: https://tierracolombiana.org/comunas-de-bucaramanga/

Con relación a la topografía, se encuentra ubicada sobre la cordillera oriental (rama de la cordillera de los Andes), es en promedio un $15 \%$ plana, $30 \%$ ondulada y el restante 55 $\%$ es quebrada. Como la mayor parte del país, las precipitaciones de la zona son de tipo bimodal (marzo-mayo y septiembre-noviembre) [12].

\section{Datos y métodos}

Teniendo en cuenta lo anterior y dado que los compuestos orgánicos volátiles (COV) (hidrocarburos en estado gaseoso a la temperatura ambiente normal los cuales son muy volátiles a dicha temperatura) se liberan durante la quema de combustibles como la gasolina, el transporte vehicular es la fuente de emisión que afecta significativamente a la comunidad. Dado que las motos y los autos particulares son los que más contribuyen a la emisión de COV, se midieron los aforos para las tres franjas horarias de mayor flujo vehicular en la zona, en el punto de mayor concentración correspondiente a la parada de bus dado que en el momento del arranque se produce la mayor liberación de contaminantes, con el fin de obtener los valores de los COV liberados y del PM2,5 más altos posibles para lo cual se establecieron las siguientes categorías: Autos (vehículos 
livianos se incluirán colectivos), Buses (se incluirán todos los tipos de servicio incluidas las busetas y alimentadores), Camiones (se incluirán todos los tipos de camiones), Motos (vehículos tipo motocicleta y triciclo auto propulsados). Se utilizó el método de observación y conteo diario de vehículos y se tomaron registros en el mismo lugar, fechas y franjas horarias de las demás mediciones: tres veces al día (en las franjas de horas pico 7-8, 12-13 y 16-17 horas) de lunes a sábado durante 26 días (octubre - noviembre de 2017) y la recopilación de información se realizó en el programa Excel. Los valores promedio de los 26 días en cada franja se puede ver en el Anexo $1 \mathrm{y}$ los resultados de los aforos vehiculares en el Anexo 2. Se utilizó un Multímetro manual de Calidad de aire con las siguientes características:

PM2.5: Rango: 0-500 $\mu \mathrm{g} / \mathrm{m}^{3}$; precisión: $\pm 10 \%$; ciclo de muestreo: 1 segundo, VOC: rango: 0-10 ppm; precisión: \pm 10\%; ciclo de muestreo: 1 segundo, Temperatura: Rango: 10-80 ${ }^{\circ} \mathrm{C}$ precisión: $\pm 0 . \quad 3{ }^{\circ} \mathrm{C}$; ciclo de muestreo: 2 segundos, Humedad: Rango: 0-100\% RH; precisión: $\pm 3 \%$ $\mathrm{RH}$; ciclo de muestreo: 2 segundo.

Se asume estado estable y régimen laminar (teniendo en cuenta la linealidad de la avenida en la zona de estudio y sabiendo que la magnitud de la velocidad no excede $\operatorname{los} 3 \mathrm{~m} / \mathrm{s}$ ) y no se analizan componentes individuales de los contaminantes. Se utilizó el software R (V.3.5.2) con el paquete Openair [15], especial para análisis atmosférico.

\section{Análisis de datos}

La metodología aplicada fue de tipo descriptiva y correlacional partiendo de la toma de datos necesarios para conocer las condiciones climatológicas de la zona y otras variables que permiten describir el estado de contaminación [17]. No se analizan componentes individuales de COV para ésta investigación.

La normativa recomienda una ubicación de los aparatos de medida a una altura de $10 \mathrm{~m}$ sin embargo dado que se trata de un medidor manual y el interés es evaluar la contaminación en el entorno de la universidad, se realizaron las mediciones en un segundo piso, siempre en la misma posición, por otra parte, como se ha mencionado previamente, dentro de los parámetros que actualmente mide la estación más cercana no incluye el PM2,5, relevante a la hora de hacer análisis ambientales, como principal contaminante del aire urbano en Colombia y en particular en ésta región puesto que al ser producido por la combustión de fósiles y al emitirse al ambiente se mezcla con el aire donde de acuerdo a la meteorología y la topografía de la zona presenta diferente dispersión.

Las condiciones de la zona no estaban caracterizadas por lo tanto se realizó la medición, promedio y análisis de las variables Temperatura y Humedad Relativa para definir las condiciones en la época del año del análisis (septiembreoctubre). En cuanto a la dirección y velocidad del viento, de acuerdo al Atlas del viento, en la Región Andina se identifica un patrón de comportamiento sobre las regiones de montaña, asociado al flujo típico de los vientos alisios, recurrentes durante todo el año, esto permite tomar como referente el periodo de los promedios registrado para las mediciones de la estación ubicada en Neomundo-Centro de Convenciones es de 2005-2009) el informe de calidad de aire 2010 de la CDMB teniendo en cuenta que presenta condiciones similares a la zona de interés.

El modelo estadístico aplicado se tomó de las librerías del software R dada su capacidad para hacer análisis, en este caso de tipo ambiental con su librería OpenAir. Este software es libre y de gran uso en el sector público y privado dadas sus potencialidades en análisis estadístico. Dentro de sus características se encuentran las siguientes: Permite la manipulación de datos, cálculos de modelos y el desarrollo de gráficos; es eficiente con matrices grandes y se pueden importar datos de distintos tipos de formato; es posible analizar datos estadísticamente y visualizarlos directamente.

El software R cuenta con un paquete especial para análisis ambientales que es el Openair. Este software está siendo utilizado actualmente para estudios ambientales, dentro de ello el IDEAM lo está aplicando a estudios de calidad de aire. Se realizaron corridas para caracterizar las variables y las relaciones entre ellas, con el propósito de determinar en qué medida cada clase de fuente móvil (categorías), incide en los resultados de PM2,5 y COV.

\section{Resultados}

\section{Dirección y velocidad del viento}

A partir de los datos meteorológicos registrados en el informe del IDEAM (2012) respecto al promedio multianual (2005-2009) [16], se tuvo en cuenta lo referente a la velocidad del viento en Neomundo (estación meteorológica más cercana) para los meses septiembre y octubre, donde los meses de agosto a octubre corresponden a la época con mayor velocidad del viento de acuerdo a dicho informe. Adicionalmente, en la franja del medio día son ligeramente mayores las magnitudes de velocidad; en la tarde disminuye nuevamente. El promedio para la estación Neomundo es de $0,71 \mathrm{~m} / \mathrm{s}$ y su dirección es $16^{\circ}$ (NNE), sin embargo, al analizar para las franjas seleccionadas se observa cambio en la dirección y en la velocidad del viento en dichas franjas, procedentes principalmente del norte al medio día y del noreste en la tarde como se resume en la Tabla 3.

Tabla 3. Datos de velocidad en las franjas de estudio

\begin{tabular}{ccr}
\hline $\begin{array}{c}\text { Franja } \\
\text { horaria }\end{array}$ & $\begin{array}{c}\text { Dirección de la } \\
\text { velocidad (grados) }\end{array}$ & $\begin{array}{c}\text { Magnitud de } \\
\text { la velocidad (m/s) }\end{array}$ \\
\hline $\mathbf{6 - 7}$ horas & 227,9 & 0,2 \\
\hline $\mathbf{1 2 - 1 3}$ & 7,8 & 2,9 \\
horas & & 1,3 \\
\hline $\begin{array}{c}\mathbf{1 8 - 1 9} \\
\text { horas }\end{array}$ & 50,2 & \\
\hline
\end{tabular}

Fuente: autores

El promedio de las medidas de velocidad y dirección del viento para un rango de 20 años, de acuerdo al IDEAM se muestra en la rosa de los vientos de la Fig. 3, donde se muestra que principalmente los vientos provienen del norte. 


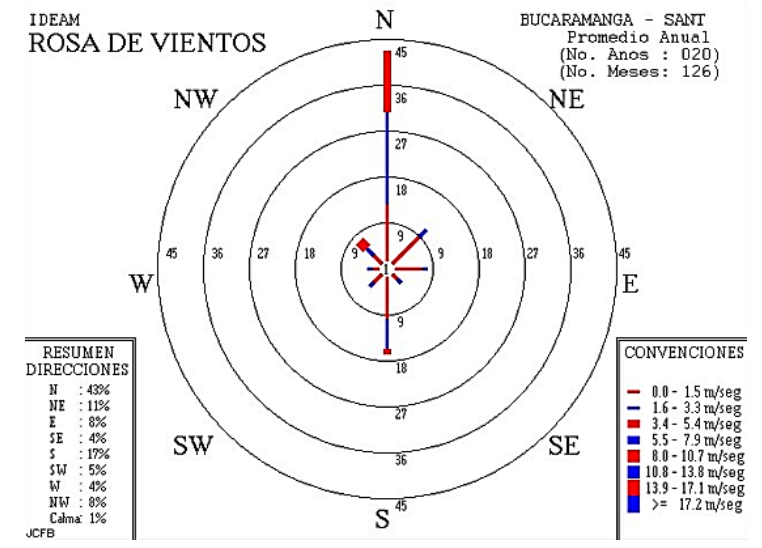

Figura 3. Rosa de los vientos Bucaramanga Fuente: Programa de meteorología aeronáutica IDEAM.

http://bart.ideam.gov.co/cliciu/rosas/viento.htm

Por tal razón para el proceso de simulación se tendrán en cuenta los tres escenarios por separados teniendo en cuenta el valor medio de concentración en dicha franja.

Aforo vehicular

Para concretar el análisis anterior, teniendo en cuenta que es una zona estudiantil, con alto flujo vehicular entre semana, los datos se totalizaron para las 3 categorías de vehículo y se agruparon en otras dos categorías: Entre semana (grupo 1) y sábados (grupo 2). Los porcentajes de acuerdo a los aforos registrados evidencian un número de vehículos ligeramente mayor el día viernes respecto al día sábado sin embargo los porcentajes entre las categorías se mantienen muy cercanos. La categoría vehicular que más se presenta diariamente corresponde a los automóviles, en seguida la categoría motos y en muy bajo porcentaje la categoría camiones y buses. En la Fig. 4 se muestra el caso del día viernes en la franja de la noche.

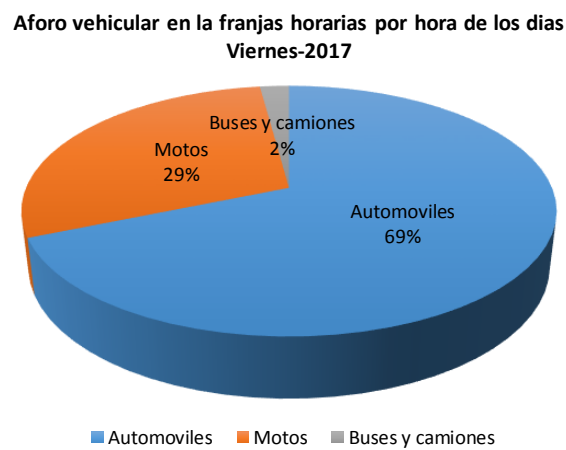

Figura 4. Composición del parque automotor promedio (día viernes en la noche) para la zona de estudio (septiembre-octubre) 2017.

Fuente: autores

Para hacer un análisis más detallado, se estipularon los escenarios por franjas horarias mostrados en la tabla 4 para realizar las corridas del modelo.

Utilizando el software R, se analizó la distribución del PM2,5 en relación con las demás variables. Con respecto a la dirección y velocidad de viento, se hicieron las corridas del modelo en cada una de las 3 franjas propuestas (mañana, tarde y noche) y en general se encontraron correlaciones con tendencias similares.

Tabla 4. Escenarios para las corridas del modelo

\begin{tabular}{|c|c|c|c|}
\hline $\begin{array}{c}\text { Franja } \\
\text { horaria }\end{array}$ & $\begin{array}{r}\text { 6-7 } \\
\text { horas }\end{array}$ & $\begin{array}{l}\begin{array}{c}12-13 \\
\text { horas }\end{array} \\
\text { hor }\end{array}$ & $\begin{array}{c}18-19 \\
\text { horas }\end{array}$ \\
\hline $\begin{array}{l}\text { Dirección } \\
\text { (grados) }\end{array}$ & 227,9 & 7,8 & 50,2 \\
\hline $\begin{array}{l}\text { Magnitud } \\
\text { de la } \\
\text { velocidad } \\
(\mathrm{m} / \mathrm{s})\end{array}$ & 0,2 & 2,9 & 1,3 \\
\hline Análisis 1 & $\begin{array}{l}\text { PM2,5 } \\
\text { y COV }\end{array}$ & $\begin{array}{l}\text { PM2,5 } \\
\text { y COV }\end{array}$ & $\begin{array}{l}\text { PM2,5 } \\
\text { y COV }\end{array}$ \\
\hline Análisis 2 & $\begin{array}{c}\text { PM2,5, } \\
\text { motos, } \\
\text { autos, } \\
\text { buses y } \\
\text { camiones }\end{array}$ & $\begin{array}{c}\text { PM2,5, } \\
\text { motos, } \\
\text { autos, } \\
\text { buses y } \\
\text { camiones }\end{array}$ & $\begin{array}{c}\text { PM2,5, } \\
\text { motos, } \\
\text { autos, } \\
\text { buses y } \\
\text { camiones }\end{array}$ \\
\hline Análisis 3 & $\begin{array}{c}\text { PM2,5, } \\
\text { humedad } \\
\text { relativa y } \\
\text { temperatura }\end{array}$ & $\begin{array}{c}\text { PM2,5, } \\
\text { humedad } \\
\text { relativa y } \\
\text { temperatura }\end{array}$ & $\begin{array}{c}\text { PM2,5, } \\
\text { humedad } \\
\text { relativa y } \\
\text { temperatura }\end{array}$ \\
\hline
\end{tabular}

Fuente: autores

A continuación, se describen los resultados para la franja de estudio 6-7 horas de la siguiente forma: en las figuras 5 (Análisis 1), 6 (Análisis 2) y 7 (Análisis 3).

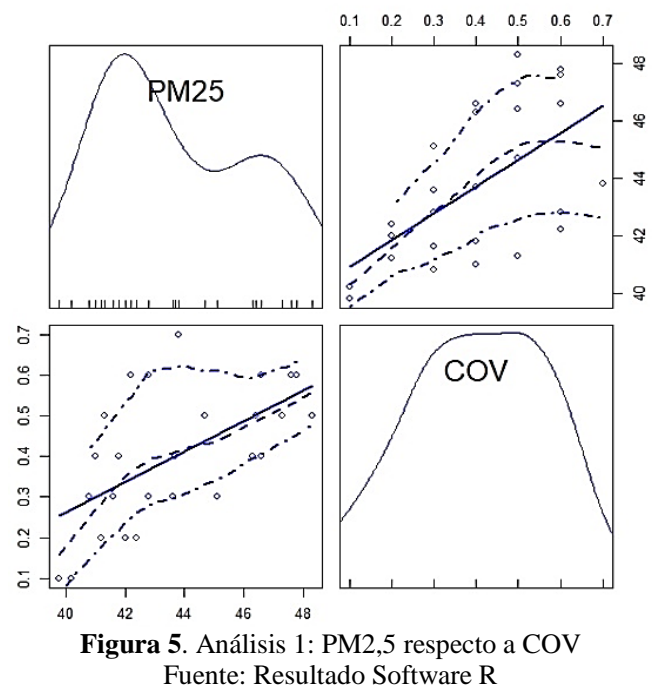

En el conjunto de gráficas de la Fig. 5 (Análisis 1), en las gráficas superior izquierda e inferior derecha, se observa la distribución es la estimación de la densidad Kernel de un conjunto de datos del PM2,5 y de los COV respectivamente. Este es un método no paramétrico que permite calcular la función de la densidad de probabilidades de una variable de la que se desconoce su distribución. En las gráficas superior derecha e inferior izquierda, se muestra la dispersión con correlación positiva, lo cual permite asociar el 
tráfico vehicular con el PM2,5: A mayor liberación de Compuestos Orgánicos Volátiles, mayor PM2,5.

En el conjunto de gráficas de la Fig. 6 (Análisis 2), en las figuras correspondientes a la diagonal (desde la superior izquierda hasta la inferior derecha), se muestra la estimación de la densidad Kernel distribución para el PM2,5 y de las tres categorías de vehículos (motos, autos, buses y camiones). En este caso, se puede ver en las demás figuras, que hay una correlación positiva con dispersiones similares al comparar los tres tipos de vehículos, por otra parte, en las gráficas de la primera fila, la figura (a) muestra un aumento significativo entre el PM2,5 y la cantidad de motos, en la figura (b) el PM2,5 y la cantidad de vehículos tiene menor dependencia y en la (c) la cantidad de buses y camiones no muestra incidencia en el PM2,5 (Se aclara que en el punto de medición solamente circula 1 ruta de metrolinea y escasos camiones). Lo anterior permite inferir que aun cuando como se evidencia en los resultados de los aforos vehiculares (Anexo 2), el número de vehículos duplica del número de motos, la cantidad de PM2,5 incrementa en mayor medida debido a las motocicletas por lo tanto son la mayor fuente de emisión entre las tres categorías comparadas (Autos, Motos, Buses y camiones). Lo anterior está acorde con los resultados reportados por el AMB donde muestran que las motos contaminan más que los automóviles [12]

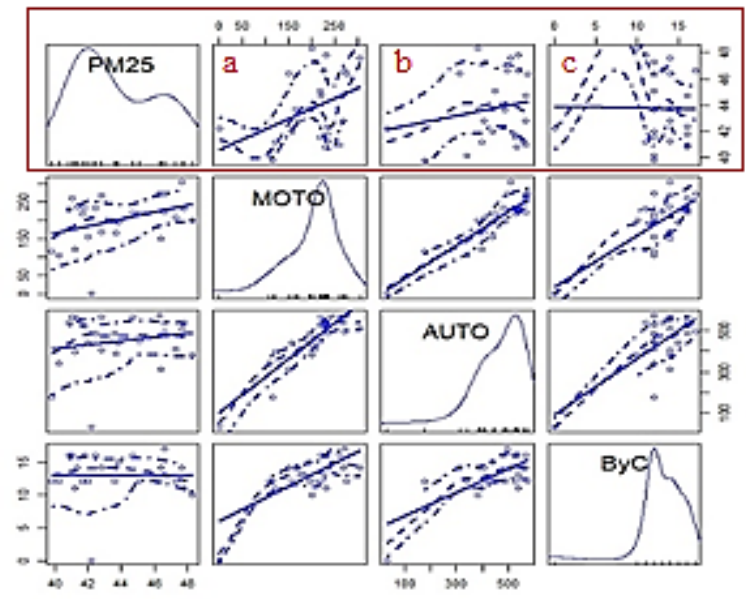

Figura 6. Análisis 2: PM2,5 respecto a vehículos a. Motos, b. Autos, c.Buses y camiones

Fuente: Resultado Software R

Las demás gráficas de la figura 6 muestran que la proporción de motos respecto a autos y respecto a buses y camiones se mantiene durante los días de medición. Por otra parte, es de interés analizar la correlación entre el PM2,5 respecto a la humedad relativa y respecto a la temperatura. La variación espacio temporal de las variables mostrada en la Fig. 6 permite tomar algunos referentes por lo tanto se recurre a la representación de la correlación que se observa en la figura 7.

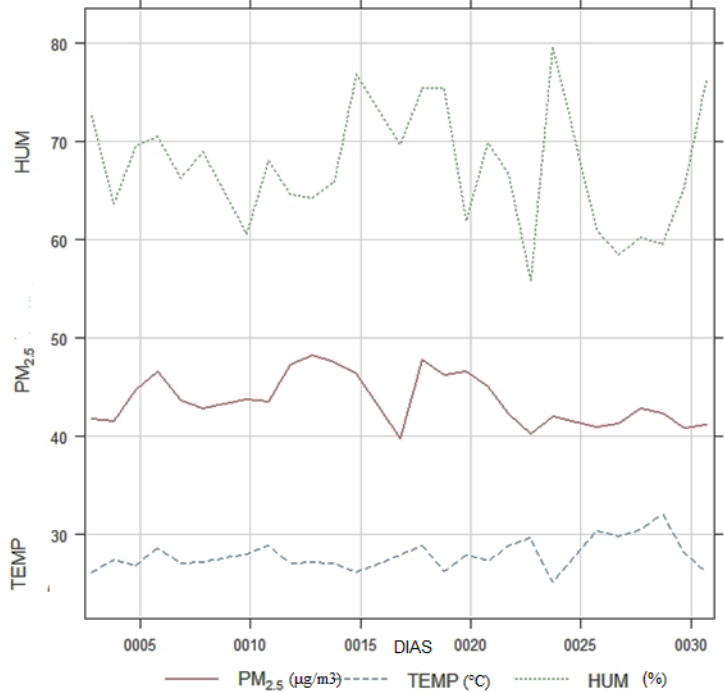

Figura 6. Gráfica espacio-temporal para los 26 días de medición de PM2.5, Humedad relativa y Temperatura

Fuente: Resultado Software R

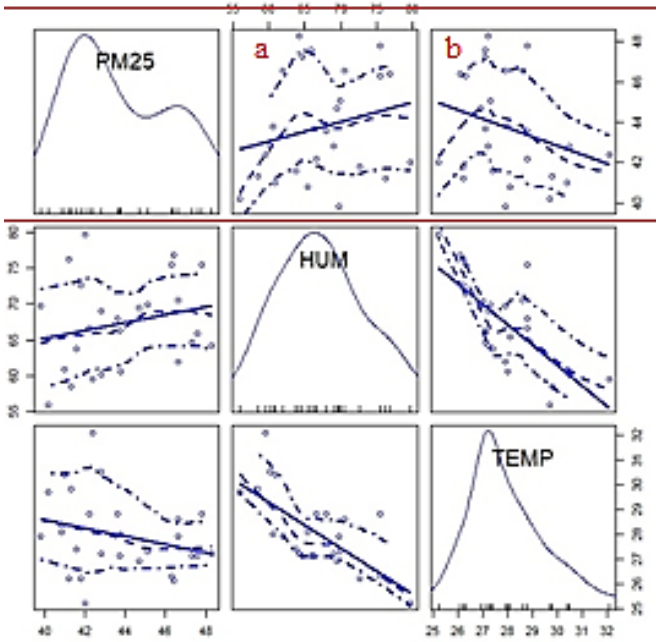

Figura 7. Análisis 3:PM2.5 respecto a: a. Humedad relativa y b.Temperatura

Fuente: Resultado Software R

En el grupo de gráficas de la Fig. 7 (Análisis 3), en las figuras correspondientes a la diagonal (desde la superior izquierda hasta la inferior derecha), se muestra la estimación de la densidad Kernel distribución para el PM2,5, Humedad Relativa y Temperatura. En las gráficas de la primera fila, la figura (a) muestra una correlación positiva del PM2,5 con la humedad relativa y la figura (b)muestra que la correlación es negativa con respecto a la temperatura; este comportamiento permite asociar la permanencia del contaminante en la atmósfera con el estado de tiempo dado que es una zona con humedad relativa alta (es decir, tendrá mayor cantidad de PM2.5 en el aire). Además, se puede evidenciar la relación inversa entre la temperatura y la humedad relativa mencionada anteriormente. 


\section{Discusión}

En la primera fase del presente estudio realizado, se encontró que la correlación entre la humedad relativa y la temperatura es negativa $(-0.78)$. También se observa una disminución significativa en el PM2,5 el día del "no carro "en la ciudad y en un lunes festivo y que a medida que las temperaturas medias diarias aumentan, también lo hacen los valores medios de PM2,5 [17]. En ésta segunda fase de análisis el interés se centra en la relación de las anteriores características respecto a la concentración de contaminantes en una franja específica (franja de la mañana) para el promedio de las mediciones (no por días).

En el caso de la relación entre PM2,5 y COV mostrado en la Fig. 4 se corrobora la dispersión con correlación positiva en dichas variables con relación a estudios como el realizado por García [5], lo cual permite asociar el tráfico vehicular con el PM2,5: A mayor liberación de Compuestos Orgánicos Volátiles, mayor PM2,5.

Adicionalmente, el resultado de la correlación entre el PM2,5 y el tipo de vehículo de la Fig. 5 muestra una mayor dependencia entre el PM2,5 y las motocicletas, en segundo lugar, la dependencia con los automóviles es menor y en tercer lugar con los Buses y camiones. El resultado sugiere que de las tres categorías (Motos, Automóviles, Buses y camiones), las motos contaminan más (cabe resaltar que, en el aforo vehicular, el número de automóviles superan el doble del número de vehículos según lo muestra la Fig. 3). El resultado concuerda tanto con el resultado de la Tabla 2. Del informe del AMB, como con la conclusión del estudio comparativo realizado en Bogotá con relación a la contaminación (PM entre otros) producida por una motocicleta con motor de cuatro tiempos $(150 \mathrm{cc})$ y por un vehículo a gasolina $(1500 \mathrm{cc})$, realizado por Cesvi Colombia y su departamento de Investigación quienes al comparar sus gases de escape encontraron que la motocicleta supera los valores de emisión de VOC, CO y PM con respecto al automóvil [18].

\section{Conclusiones}

La contaminación por material particulado es de suma importancia, y en especial el PM2,5, el cual ha empezado a medirse en algunos puntos de la ciudad posterior a la presente investigación, por tanto, esta medición inicial aporta un escenario para el periodo septiembre-octubre, que puede considerarse un punto de partida para análisis posteriores de calidad de aire en zonas cercanas a la universidad, teniendo en cuenta que el análisis se centra en datos de menos de 1 mes (6 días durante 4 semanas aproximadamente) que no han sido registrados de manera puntual en la zona de interés (zona estudiantil). Por otra parte, Este tipo de análisis puede ser aplicado a otros contaminantes de interés siempre y cuando se conozcan las características de la zona por lo tanto se convierte en una herramienta económica y útil para análisis en zonas donde no es posible tener estaciones meteorológicas de las entidades ambientales dado su alto costo. Lo anterior facilita crear desarrollos que permitan mejorar la calidad de vida de quienes residen en su cercanía.

El análisis de la dispersión de contaminantes en la zona de interés muestra que efectivamente el PM2,5 está asociado a los COV, que pueden ser en su mayoría provenientes del combustible generado por las fuentes móviles que circulan por la vía dada la alta correlación encontrada en los análisis. Teniendo en cuenta los resultados anteriores y los resultados estadísticos del Anexo 3, con relación a la variable temperatura se observa que al medio día sube en $3{ }^{\mathrm{a}} \mathrm{C}$ respecto a la mañana y disminuye la humedad relativa en un 7\%. Los resultados promedio de todas las mediciones de PM2,5 y $\mathrm{COV}$ por franja se mantienen posiblemente debido a que la velocidad del viento proveniente del Norte aumenta respecto al valor de la mañana (de $0,2 \mathrm{~m} / \mathrm{s}$ a $2,9 \mathrm{~m} / \mathrm{s}$ ) y arrastra el material particulado.

Por otra parte, es relevante el resultado de la relación del PM2,5 con la humedad relativa la cual presenta valores altos en la franja de la mañana y noche respecto al medio día. Esta relación sugiere la persistencia de los contaminantes en el ambiente por más tiempo.

En general, entre los distintos tipos de vehículos (sin tener en cuenta años ni modelos), no se observan cambios en la correlación lo cual sugiere que la relación con el PM2,5 obedece más a la cantidad de vehículos y se incrementa respecto al número de vehículos, lo cual es concordante con el análisis anterior es decir que al aumentar las medidas de aforos vehiculares aumenta la cantidad de COV y de PM2,5 registrados. Se observan cambios menores en la dispersión cuando se analizan los tres tipos de vehículo, posiblemente debido a características como tipo de combustible entre otras. Se aclara que, para el presente estudio, en las categorías estipuladas no se tuvieron en cuenta características particulares (combustible, año, cilindraje, etc.)

\section{Reconocimientos}

Agradecimiento especial a la Dirección de Investigaciones de la UNAB, por la financiación de esta investigación.

\section{Referencias}

[1] Herrera, V., Rodríguez, L., \& Mágara, K.. Concentraciones de material particulado fino en microambientes comunitarios e intradomiciliarios en Bucaramanga, Colombia. Revista del Observatorio de Salud Pública de Santander, Año 11. No.1. (2016) 3-12. http://observatorio.co/web/publicaciones/calidad_aire_bucaramanga_ material particulado microambientes comunitarios intradomiciliari os.pdf

[2] SIAC. (s.f.). http://www.siac.gov.colemisionesaire.

[3] Morales, T., \& Arias, J. Contaminación vehicular en la conurbación Pereira-Dosquebradas. Luna Azul, (2013).101-129. http://www.scielo.org.co/scielo.php?pid=S1909$24742013000200009 \&$ script $=$ sci abstract\&tlng=es.

[4] Ramírez, O., Sánchez, A., Amato, F., Catacolì, R., Rojas, N., \& De la Rosa, J. Chemical composition and source apportionment of PM10 at an urban background site in a high-altitude Latin American megacity (Bogota, Colombia). Environmental Pollution, Vol.233 (2018). (142155) https://doi.org/10.1016/j.envpol.2017.10.045. 
[5] García, N. E. Niveles de material particulado en la zona urbana de Cuenca-Ecuador y su relación con el tráfico vehicular. Revista Latinoamericana el Ambiente y las Ciencias, 9(20): (2018). 41-55 http://dspace.ucuenca.edu.ec/bitstream/123456789/30540/2/document o.pdf.

[6] Granada, L. C. Estimación de las emisiones de fuentes móviles usando el mobile 6. AVANCES Investigación en Ingeniería - 2007 No. 6, (2007).17-29. http://www.unilibre.edu.co/revistaavances/avances6/r6 art2.pdf.

[7] Quijano, A., Quijano, M., \& Meléndez, I. Influencia de la combustión vehicular en la calidad del aire de Pamplona - Colombia. BISTUA; Revista de la Facultad de Ciencias Básicas, V.14 fasc1 (2016). p.1731.

http://revistas.unipamplona.edu.co/ojs_viceinves/index.php/BISTUA/ article/view/1929.

[8] Buitrago., J. Aplicación del modelo Gaussiano para determinar la calidad de aire de Manizales. Manizales: Tesis. (2003). http://bdigital.unal.edu.co/969/1/jaimehernanbuitragoarango.2003.pdf

[9] Arrieta, A. Dispersión de material particulado (PM 10), con interrelación de factores meteorológicos y topográficos. Ingeniería Investigación $Y$ Desarrollo, 16(2), (2016). 43-54. https://doi.org/10.19053/1900771X.v16.n2.2016.5445.

[10] Arciniegas, C. Diagnóstico Y Control De Material Particulado: Partículas Suspendidas Totales Y Fracción Respirable PM10. Revista
Luna
Azul,
(34),
(2012).
195-213

http://www.scielo.org.co/scielo.php?pid=S1909-

$24742012000100012 \&$ script $=$ sci $\quad$ abstract\&tlng $=$ pt.

[11] Ministerio de Ambiente y Desarrollo Sostenible. Guía para la elaboración de inventarios de emisiones atmosféricas. Bogotá: (2017). https://www.minambiente.gov.co/images/AsuntosambientalesySector ialyUrbana/pdf/emisiones_atmosfericas_contaminantes/documentos relacionados/GUIA_PARA_LA_ELABORACION_DE_INVENTAR IOS DE EMISIONES ATMOSFERICAS.pdf.

[12] AMB. (s.f.). El AMB realiza monitoreo diario de la tendencia de la calidad del aire en Bucaramanga, Floridablanca y girón. Obtenido de https://www.amb.gov.co/el-amb-realiza-monitoreo-diario-de-latendencia-de-la-calidad-del-aire-en-bucaramanga-floridablanca-ygiron/

[13] Alcaldía de Bucaramanga. (14 de Marzo de 2020). Conozca cómo se ha comportado la calidad del aire en tres puntos de Bucaramanga. Obtenido de https://www.bucaramanga.gov.co/noticias/conozcacomo-se-ha-comportado-la-calidad-del-aire-en-tres-puntos-debucaramanga/

[14] Rodríguez, L., Castro, H. R., Herrera, A., \& Niederbacher, J. (2010). Contaminación atmosférica y sus efectos en la salud en Bucaramanga. Revista del Observatorio de Salud Pública de Santander, Año 5 No.1 3-18

[15] http://web.observatorio.co/publicaciones/revista_salud_ambiental_art iculo_principal.pdf.

[16] R-Core-Team. (2013). A language and environment for statistical. Obtenido de http://www.R-project.org/.

[17] IDEAM. Estado de la Calidad del Aire en Colombia 2007-2010. (2012). Bogotá. http://www.ideam.gov.co/documents/51310/68521396/5.+Informe+d el+estado+de+la+calidad+del+aire+2007-2010.pdf/52d841b0-afd0$4 \mathrm{~b} 8 \mathrm{e}-83 \mathrm{e} 5-444 \mathrm{c} 3 \mathrm{~d} 17 \mathrm{ed} 29$ ? version $=1.0$.

[18] Beleno, L., \& Colegial, J. (2018). Análisis de la Contaminación por flujo vehicular en un entorno universitario. BISTUA Revista de la facultad $d$ ciencias básicas, 16(1):28 a 41 DOI: https://doi.org/10.24054/01204211.v1.n1.2018.3190.

[19] ¿Cuál contamina más entre motos Vs. automóviles?. Auto Crash. Revista ediciòn digital No.54. Mayo, 2019

https://www.revistaautocrash.com/cual-contamina-mas-entre-motosvs-automoviles/ 
ANEXO 1. Muestra promedio de las Mediciones de PM2.5, COV, temperatura y humedad relativa para las 3 franjas horarias (horas pico medidas de lunes a sábado durante 26 días)

\begin{tabular}{|c|c|c|c|c|c|c|}
\hline dias:26 & \multicolumn{2}{|c|}{ Promedios 7:00-7:55 } & \multicolumn{2}{|c|}{ Promedios 12:00-12:55 } & \multicolumn{2}{|c|}{ Promedios 18:00-18:: } \\
\hline PM 2,5 ( $\mu \mathrm{g} / \mathrm{m} 3)$ & \multirow{4}{*}{ 7:00 } & 44,3 & \multirow{4}{*}{$12: 00$} & 44,5 & \multirow{4}{*}{$18: 00$} & 45,4 \\
\hline (COV) PPM & & 0,5 & & 0,5 & & 0,6 \\
\hline TEMP. $(\stackrel{\circ}{ } \mathrm{C})$ & & 28,1 & & 31,2 & & 28,3 \\
\hline HUM-REL (\%) & & 66,9 & & 60,1 & & 71,0 \\
\hline PM 2,5 $(\mu \mathrm{g} / \mathrm{m} 3)$ & \multirow{4}{*}{ 7:05 } & 44,6 & \multirow{4}{*}{ 12:05 } & 43,9 & \multirow{4}{*}{$18: 05$} & 44,1 \\
\hline (COV) PPM & & 0,6 & & 0,6 & & 0,8 \\
\hline TEMP.(으) & & 28,0 & & 30,6 & & 27,3 \\
\hline HUM-REL (\%) & & 68,8 & & 60,0 & & 71,4 \\
\hline PM 2,5 $(\mu \mathrm{g} / \mathrm{m} 3)$ & \multirow{4}{*}{ 7:10 } & 44,2 & \multirow{4}{*}{$12: 10$} & 43,5 & \multirow{4}{*}{$18: 10$} & 44,6 \\
\hline (COV) PPM & & 0,5 & & 0,4 & & 0,6 \\
\hline TEMP.(으) & & 27,7 & & 30,9 & & 28,3 \\
\hline HUM-REL (\%) & & 68,8 & & 60,1 & & 70,2 \\
\hline PM 2,5 $(\mu \mathrm{g} / \mathrm{m} 3)$ & \multirow{4}{*}{$7: 15$} & 44,1 & \multirow{4}{*}{$12: 15$} & 44,4 & \multirow{4}{*}{$18: 15$} & 44,6 \\
\hline (COV) PPM & & 0,4 & & 0,5 & & 0,4 \\
\hline TEMP. $\left({ }^{\circ} \mathrm{C}\right)$ & & 27,7 & & 29,8 & & 28,1 \\
\hline HUM-REL (\%) & & 69,7 & & 60,5 & & 70,1 \\
\hline PM 2,5 $(\mu \mathrm{g} / \mathrm{m} 3)$ & \multirow{4}{*}{$7: 20$} & 43,5 & \multirow{4}{*}{$12: 20$} & 43,9 & \multirow{4}{*}{$18: 20$} & 44,3 \\
\hline \begin{tabular}{|c|} 
(COV) PPM \\
\end{tabular} & & 0,5 & & 0,4 & & 0,4 \\
\hline TEMP. $\left({ }^{\circ} \mathrm{C}\right)$ & & 27,7 & & 30,9 & & 28,0 \\
\hline HUM-REL (\%) & & 66,1 & & 61,0 & & 70,8 \\
\hline $\mathrm{PM} 2,5(\mu \mathrm{g} / \mathrm{m} 3)$ & \multirow{4}{*}{$7: 25$} & 43,9 & \multirow{4}{*}{$12: 25$} & 43,7 & \multirow{4}{*}{$18: 25$} & 45,3 \\
\hline \begin{tabular}{|c|} 
(COV) PPM \\
\end{tabular} & & 0,4 & & 0,5 & & 0,4 \\
\hline TEMP.(으) & & 27,8 & & 31,1 & & 27,8 \\
\hline HUM-REL (\%) & & 67,5 & & 61,2 & & 69,7 \\
\hline PM 2,5 $(\mu \mathrm{g} / \mathrm{m} 3)$ & & 44,1 & & 44,3 & & 43,3 \\
\hline \begin{tabular}{|c|} 
(COV) PPM \\
\end{tabular} & $7 \cdot 30$ & 0,3 & $12 \cdot 30$ & 0,3 & 18.30 & 0,4 \\
\hline TEMP.(ํ) & 1.50 & 27,8 & 12.30 & 31,3 & 10.00 & 27,9 \\
\hline HUM-REL (\%) & & 67,2 & & 59,2 & & 71,6 \\
\hline $\mathrm{PM} 2,5(\mu \mathrm{g} / \mathrm{m} 3)$ & & 43,5 & & 43,8 & & 43,9 \\
\hline (COV) PPM & 7.35 & 0,4 & 12.35 & 0,3 & 18.32 & 0,6 \\
\hline TEMP. $(\stackrel{\circ}{ } \mathrm{C})$ & & 27,9 & & 31,0 & & 28,0 \\
\hline HUM-REL (\%) & & 66,9 & & 59,0 & & 71,9 \\
\hline $\mathrm{PM} 2,5(\mu \mathrm{g} / \mathrm{m} 3)$ & & 43,4 & & 44,1 & & 44,0 \\
\hline (COV) PPM & $7: 40$ & 0,3 & $12 \cdot 40$ & 0,3 & 18.40 & 0,4 \\
\hline TEMP. $(\stackrel{\circ}{ } \mathrm{C})$ & & 28,1 & 12.40 & 31,1 & 10.40 & 27,9 \\
\hline HUM-REL (\%) & & 66,5 & & 58,7 & & 71,5 \\
\hline PM 2,5 $(\mu \mathrm{g} / \mathrm{m} 3)$ & & 43,1 & & 43,4 & & 43,4 \\
\hline \begin{tabular}{|c|} 
(COV) PPM \\
\end{tabular} & 7.45 & 0,3 & 12.45 & 0,3 & 18.45 & 0,3 \\
\hline TEMP. $(\stackrel{\circ}{ } \mathrm{C})$ & & 28,2 & 12.75 & 30,8 & 10.75 & 28,0 \\
\hline HUM-REL (\%) & & 66,2 & & 59,6 & & 71,0 \\
\hline $\mathrm{PM} 2,5(\mu \mathrm{g} / \mathrm{m} 3)$ & & 43,5 & & 43,3 & & 42,9 \\
\hline \begin{tabular}{|c|} 
(COV) PPM \\
\end{tabular} & 7.50 & 0,3 & 12.50 & 0,2 & 18.50 & 0,2 \\
\hline TEMP.(으) & 1.50 & 28,3 & 12.50 & 30,9 & 10.50 & 28,0 \\
\hline HUM-REL (\%) & & 65,6 & & 60,0 & & 71,2 \\
\hline $\mathrm{PM} \mathrm{2,5}(\mu \mathrm{g} / \mathrm{m} 3)$ & & 43,2 & & 42,9 & & 43,2 \\
\hline (COV) PPM & $7 \cdot 55$ & 0,3 & 12:55 & 0,3 & $18: 55$ & 0,2 \\
\hline TEMP. $\left({ }^{\circ} \mathrm{C}\right)$ & נכ. & 28,5 & נI.J. & 31,0 & I0.J & 27,9 \\
\hline HUM-REL (\%) & & 65,1 & & 60,0 & & 70,3 \\
\hline
\end{tabular}


ANEXO 2 Aforos vehicular medido para las 3 franjas horarias (horas pico)

\begin{tabular}{|c|c|c|c|c|c|}
\hline viernes 2017 & & sábado 2017 & & \begin{tabular}{|l|} 
VIERNES porcentaje \\
por categoria
\end{tabular} & $\begin{array}{l}\text { SABADO porcentaje } \\
\text { por categoria }\end{array}$ \\
\hline 7.8 HORAS & Veh ${ }^{*} H$ & 7-8 HORAS & $\operatorname{Veh}^{*} \mathrm{H}$ & & \\
\hline Automoviles & 444 & Automoviles & 388 & 67.1 & 70.5 \\
\hline \begin{tabular}{|l|l|} 
Matos \\
\end{tabular} & 204 & Motos & 149 & 30.8 & 27.1 \\
\hline \multirow[t]{2}{*}{ Buses ycamiones } & 14 & \begin{tabular}{|l} 
Buses $y$ camiones \\
\end{tabular} & 13 & 2.1 & 2.4 \\
\hline & 662 & Total & 550 & & \\
\hline 12-13 HORAS & \multirow{2}{*}{ Ven ${ }^{*} H$} & 12-13 HORAS & $\operatorname{Veh}^{*} \mathrm{H}$ & & \\
\hline & & & & & \\
\hline \begin{tabular}{|l|l} 
Automoviles \\
\end{tabular} & 495 & Automoviles & 469 & 68.6 & 71.3 \\
\hline \begin{tabular}{|l|l|} 
Motos \\
\end{tabular} & 211 & Motos & 175 & 29.2 & 26.6 \\
\hline \multirow[t]{2}{*}{ Busesy ycamiones } & 16 & Buses $y$ camiones & 14 & 2.2 & 2.1 \\
\hline & 722 & Total & 658 & & \\
\hline 18-19 HORAS & \multirow{2}{*}{ ven ${ }^{*} H$} & 18-19 HORAS & $\operatorname{Ven}^{*} \mathrm{H}$ & & \\
\hline & & & & & \\
\hline Automoviles & 479 & Automoviles & 513 & 68.7 & 68.9 \\
\hline Motos & 203 & Motos & 218 & 29.1 & 29.3 \\
\hline \multirow[t]{2}{*}{ Buses ycamiones } & 15 & Buses $y$ camiones & 14 & 2.2 & 1.9 \\
\hline & 697 & Total & 745 & 100 & 100 \\
\hline Automoviles (prom) & 473 & & 457 & 68 & 70 \\
\hline \begin{tabular}{|l|} 
Motos (Promedio) \\
\end{tabular} & 206 & & 181 & 30 & 28 \\
\hline Busesy camiones (Prom) & 15 & & 14 & 2 & 2 \\
\hline
\end{tabular}


ANEXO 3. Resultados estadísticos de las mediciones

Los resultados para la franja 7-8 horas son los siguientes:

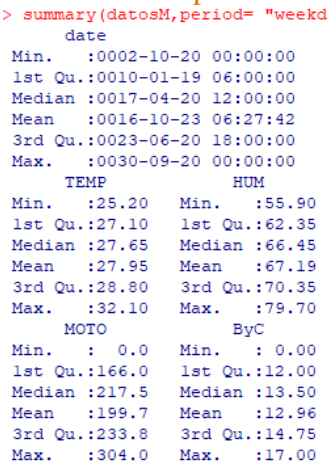

Los resultados para la franja 12-13 horas son los siguientes:

> summary (datos $\mathrm{T}$, period= "weekday")

\begin{tabular}{|c|c|c|c|c|c|c|c|}
\hline \multicolumn{3}{|c|}{ date } & \multicolumn{2}{|c|}{ weekday } & hour & \multicolumn{2}{|c|}{ PM25 } \\
\hline Min. & :0002-10- & $-2000: 00: 00$ & Jueves & $: 5$ & $0: 26$ & Min. & $: 39.50$ \\
\hline lst Qu. & $.0010-01-$ & $-19 \quad 06: 00: 00$ & Lunes & $: 4$ & & lst Qu. & $: 41.30$ \\
\hline Median & :0017-04- & $-20 \quad 12: 00: 00$ & Martes & $: 4$ & & Median & $: 43.75$ \\
\hline Mean & $: 0016-10$ & $-23 \quad 06: 27: 42$ & Miércoles & & & Mean & $: 43.77$ \\
\hline 3rd Qu. &.$: 0023-06-$ & $-20 \quad 18: 00: 00$ & Sábado & $: 4$ & & 3rd Qu. & $: 46.35$ \\
\hline Max. & :0030-09- & $-20 \quad 00: 00: 00$ & Viernes & $: 5$ & & Max. & $: 47.60$ \\
\hline & & TEMP & & GUM & & ws & wc \\
\hline Min. & $: 0.1000$ & Min. $\quad: 27.00$ & Min. & $: 48.00$ & Min. & $: 2.9$ & Min. \\
\hline lst Qu. & .0 .2250 & lst Qu.: 29.02 & 1st Qu & 1. $: 54.70$ & lst 0 & Qu.: 2.9 & 1st Qu. : \\
\hline Median & $: 0.4000$ & Median $: 30.65$ & Median & $: 59.15$ & Media & an $: 2.9$ & Median : \\
\hline Mean & $: 0.3923$ & Mean $: 30.91$ & Mean & $: 60.10$ & Mean & $: 2.9$ & Mean : \\
\hline 3rd Qu. &.$: 0.5000$ & 3rd Qu.: 32.27 & 3rd Qu & 2. $: 63.70$ & $3 \mathrm{rd} 0$ & 2u.: 2.9 & 3rd Qu.: \\
\hline Max. & $\begin{array}{l}: 0.8000 \\
\text { TOS }\end{array}$ & $\begin{array}{c}\text { Max. }: 35.00 \\
\text { MOTOS }\end{array}$ & $\begin{array}{l}\text { Max. } \\
\text { BUS }\end{array}$ & $\begin{array}{l}: 86.70 \\
\text { syc }\end{array}$ & Max. & $: 2.9$ & Max. : \\
\hline Min. & $: 191.0$ & Min. : 5.0 & Min. & $: 12.00$ & & & \\
\hline lst Qu. &.$: 492.8$ & lst Qu.: 193.5 & lst Qu. & $: 14.00$ & & & \\
\hline Median & $: 532.5$ & Median :234.0 & Median & $: 15.00$ & & & \\
\hline Mean & $: 537.7$ & Mean :218.5 & Mean & $: 14.96$ & & & \\
\hline 3rd Qu. &.$: 645.0$ & 3rd Qu.: 254.0 & 3rd Qu. & $: 16.00$ & & & \\
\hline Max. & $: 715.0$ & Max. $: 291.0$ & Max. & $: 19.00$ & & & \\
\hline
\end{tabular}

Los resultados para la franja 18-19 horas son los siguientes:

$>$ summary (datosN, period $=$ "weekday")

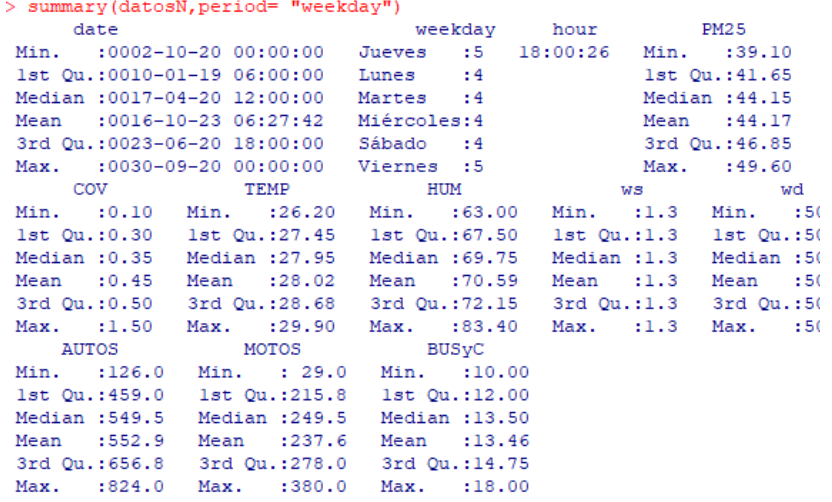

